\title{
The COVID-19 Outbreak: An Experience for Iranian Health Systems and Orthopedics Association
}

\author{
Ali Tabrizi, MD ${ }^{1 *}$; Ahmadreza Afshar, MD ${ }^{1}$ \\ ${ }^{1}$ Department of Orthopedics, Imam Khomeini Hospital, Urmia University of Medical Sciences, Urmia, Iran
}

\section{Dear Editor,}

Today, we are facing a pandemic caused by the widespread transmission of the coronavirus disease 2019 (COVID-19) in Iran and worldwide. In general, different parts of the health system, including emergency departments, intensive care units (ICUs) and outpatient clinics, face a large number of patients with acute shortterm respiratory problems leading to serious shortage of beds in outpatient departments and ICUs. ${ }^{1}$ In late 2009, following the outbreak of influenza H1N1 in the United States, a special committee was established to decide on resource management, triage, and management of patients in crises, which has played a key role in controlling and managing patients in crisis by setting the standards of crisis care for use in disasters. ${ }^{2}$ In general, health security is one of the most basic elements of a health system. The concept of health security against pandemic diseases was already clear in the 14 th century when quarantine was used to prevent the spread of pandemics and it was for the first time effective in controlling the bubonic plague. Following the increase in trade and communication between countries, health threats from infectious diseases, such as the plague and three infectious diseases caused by cholera, smallpox and yellow fever, emerged in human societies. ${ }^{2}$ Today, threats caused by viruses have created a new spectrum of infectious disease crises that require planning and preparation of health systems in different countries based on their available capabilities and facilities. Today, the global health system is facing a new crisis caused by respiratory infections. The first case of COVID-19 was reported in December 2019 in Wuhan, China. ${ }^{3}$ In Iran, the first cases of infection were officially reported on February 19, 2020. To date, 75000 acute respiratory infections caused by COVID-19 and 4500 deaths have been reported in Iran (https://www.worldometers.info/ coronavirus). ${ }^{1}$ Nearly 100 health care workers have died from acute respiratory infections - a major challenge for Iran's healthcare system over the past century. ${ }^{1}$ In Singapore, the first case was identified on January 23, 2020, and on February 7, 2020, rapid initial measures were developed and taken by the Ministry of Health to control the disease based on the orange level of the Disease Outbreak Response System Condition (DORSCON). ${ }^{3}$ This protocol was used in all public hospitals and medical departments based on the risk level, followed by a 14-day quarantine, which led to strict control and reduction of mortality. The respiratory syndrome epidemic caused by COVID-19 may not be associated with orthopedics. ${ }^{3}$ However, due to the large number of traumatic patients in Iran and general hospitals, the involvement of orthopedic surgeons and traumatic patients is inevitable. ${ }^{4}$ Therefore, the first professional guideline to control COVID-19 was developed by the Iranian Orthopedic Association (IOA) on April 15, 2020. Although orthopedic surgeons are not at the front-line of the defenders against the COVID-19 epidemic, the Iranian orthopedic community, as part of the national health system, feels deeply responsible to take part in the battle against the new coronavirus pandemic. Therefore, a comprehensive guideline was developed for all Iranian orthopedic surgeons in both Persian and English languages. Based on this guideline, five key points have been considered, including: (a) Reducing morbidity and mortality of the people in need for orthopedic care while preserving healthcare resources; (b) Minimizing disease transmission by postponing non-essential procedures and reducing face-to-face encounters in line with social distancing recommendations during this stage of the epidemic; (c) Protecting healthcare personnel; (d) Preserving feasibility and healthcare system functionality; and (e) Publishing up-to-date information for IOA members and public regarding the ongoing pandemic. ${ }^{4}$ In general, three guidelines have been developed for orthopedic surgeons regarding patient management in the COVID-19 epidemic and pandemic, and the guideline of the Iranian Orthopedic Association is one of the most complete, written in 17 pages. ${ }^{4}$ Another guideline was published on March 20, 2020 by the British Orthopedic Association which is written in 6 pages. ${ }^{5}$ According to this guideline, four goals have been considered including: providing support and reassurance to clinicians, paying attention to the limitations of efficient human resources, keeping the key resource of personnel operational, 
and triaging and contracting the service as physical and personnel resources diminish. ${ }^{5}$ A clinical guide for management of major trauma patients during the coronavirus pandemic has been compiled by England's National Health Service (NHS) on March 26, $2020 .^{6}$ This guideline focuses on management and treatment of trauma patients during the COVID-19 epidemic. ${ }^{6}$ The development of IOA guideline was an appropriate and effective measure in treatment and management of traumatic patients and improving the protection of orthopedic surgeons by providing instructions on facing with COVID-19 cases. Therefore, the comprehensive and accurate planning by the Iranian Orthopedic Association for crises and faster performance while facing crises should be considered in the future.

\section{Authors' Contribution}

Both authors contributed equally to this work.

\section{Conflict of Interest Disclosures}

None.

Ethical Statement

Not applicable.

\section{References}

1. Takian A, Raoofi A, Kazempour-Ardebili S. COVID-19 battle during the toughest sanctions against Iran. Lancet. 2020;395(10229):1035-6. doi: 10.1016/S01406736(20)30668-1.

2. Heymann DL, Chen L, Takemi K, Fidler DP, Tappero JW, Thomas MJ, et al. Global health security: the wider lessons from the west African Ebola virus disease epidemic. Version 2. Lancet. 2015;385(9980):1884-901. doi: 10.1016/S01406736(15)60858-3.

3. Chang Liang Z, Wang W, Murphy D, Po Hui JH. Novel coronavirus and orthopaedic surgery: early experiences from Singapore. J Bone Joint Surg Am. 2020;102(9):745-9. doi: 10.2106/JBJS.20.00236.

4. Iranian Orthopedics Association (IOA) Response Guidance to COVID-19 Pandemic. Education Committee of the IOA; 2020. Available from: https://iranoa.org/.

5. Management of patients with urgent orthopaedic conditions and trauma during the coronavirus pandemic; 2020. Available from: https://www.england.nhs.uk/coronavirus/secondarycare/other-resources/specialty-guides/.

6. Clinical guide for the management of major trauma patients during the coronavirus pandemic; 2020. Available from: https://www.england.nhs.uk/coronavirus/secondary-care/ other-resources/specialty-guides/. 